\title{
Exhalation Air Fungal Culture of Mechanically Ventilated Aspergillosis Suspected Patients in Concordance with Other Conventional Diagnostic Techniques
}

\author{
M Bassam Aboul-Nasr ${ }^{1 *}$, Abdel-Nasser Zohri ${ }^{2}$, Mohamed Adam ${ }^{3}$ and Enas Mahmoud Amer ${ }^{2}$ \\ ${ }^{1}$ Department of Botany and Microbiology, Faculty of Science, Sohag University, Egypt \\ ${ }^{2}$ Department of Botany and Microbiology, Faculty of Science, Assiut University, Egypt \\ ${ }^{3}$ Department of Chest Diseases and Tuberculosis, Faculty of Medicine, Assiut University, Egypt
}

*Corresponding author: M Bassam Aboul-Nasr, Department of Botany and Microbiology, Faculty of Science, Sohag University, Egypt, Tel: 01007221923; E-mail: mbassamaboulnasr@yahoo.com

Received date: July 20, 2017; Accepted date: October 11, 2017; Published date: October 18, 2017

Copyright: (c) 2017 Aboul-Nasr MB, et al. This is an open-access article distributed under the terms of the Creative Commons Attribution License, which permits unrestricted use, distribution, and reproduction in any medium, provided the original author and source are credited.

\begin{abstract}
Aspergillosis remains one of the most challenging areas of illness. It has recently emerged as a world-wide health care problem, owing to extensive use of broad spectrum antibiotics, immunosuppressive agents and increasing population of terminally ill and debilitated patients. The ultimate aim of this investigation is to minimize morbidity and mortality of mechanically ventilated aspergillosis suspected patients, who have been notoriously difficult to be diagnosed and treated, by developing an accurate and easy diagnostic tool comparable to other conventional methods. Two hundred twenty seven suspected aspergillosis patients were screened for fungal infection using different conventional tools to evaluate different respiratory specimens sputum, bronchoalveolar lavage, plural fluid, blood, biopsy and ELISA technique for galactomannan and (1-3)- $\beta$-D-glucan antigens in serum and bronchoalveolar lavage. Four Aspergillus species ( $A$. niger, A. fumigatus, A. flavus and A. terreus) were isolated and identified morphologically and genetically using the conventional diagnostic tools from suspected patients in all positive cultures. The results of the exhalation air of twenty three mechanically ventilated aspergillosis suspected patients came in accordance with those of the conventional methods used. Thus, exhalation air culture is an easily performed bedside tool for diagnosis of aspergillosis in ventilated patients.
\end{abstract}

Keywords: Aspergillosis; Aspergillus species; Diagnostic tools

\section{Introduction}

The problem of fungal infections became serious in 1960s, when wide-spectrum antibiotics and cytostatic agents entered widespread use [1]. Aspergilli cause a wide range of human ailments depending on the immune status of the host [2]. Aspergillus infection is the commonest invasive fungal infection which involves respiratory tract $[3,4]$. Among the human pathogenic species of Aspergillus, $A$. fumigates is the primary causative agent of human infections, followed by $A$. flavus, $A$. terreus, $A$. niger, and the model organism, $A$. nidulans (Emericella nidulans) [2,5]. Aspergillosis developed widely in developing countries that are home to around five-sixth of the world's population and located in the tropical and sub-tropical regions of Asia, Africa and South and Central America where fungi thrive well in the hot and humid climate [6]. There was a $35.7 \%$ increase in mortality caused by invasive aspergillosis between 1980 and 1997. The clinical symptoms and signs associated with invasive pulmonary aspergillosis are notoriously vague. An accurate diagnosis of invasive aspergillosis is important for clinical reasons; an earlier diagnosis is associated with improved patient survival and tests with a high negative predictive value may allow expensive and potentially toxic antifungal drugs to be withheld [7]. Diagnosis is based on a combination of clinical risks, symptoms and signs, culture, histopathology and detection of the fungal components such as the antigen galactomannan and direct surface analysis bymatrix-assisted laser desorption ionization time-offlight mass spectrometry (MALDI0) [8]. Radiology also plays an important role in diagnosis and follow-up. Early findings on a computed tomography (CT) of invasive pulmonary aspergillosis are ground-glass attenuation surrounding a pulmonary nodule, the halo sign [9].

The ultimate aim of this study is to minimize morbidity and mortality in mechanically ventilated aspergillosis patients, which has been notoriously difficult to be diagnosed and treated, by developing an accurate and easy diagnostic tool for them comparable to other conventional tools.

\section{Material and Methods}

\section{Mycological assay}

\section{Conventional methods:}

1. Sputum specimens: Sputum specimens were collected from the patients in sterile cubs. Five $\mathrm{ml}$ of Sabouraud dextrose broth medium were added to each specimen and shacked for one minute on vortex and incubated at $37 \pm 2^{\circ} \mathrm{C}$ for two hours to be liquefied.

2. Bronchoalveolar lavage (BAL): Bronchoscopy was done according to BTS guideline 2013 for diagnostic flexible bronchoscopy in adults.

3. Plural fluid: Plural fluid was aspirated by thoracocentesis under aseptic condition.

One $\mathrm{ml}$ from each (prepared sputum, bronchoalveolar lavage, plural fluid) specimens were transferred aseptically into three sterilized nine 
Page 2 of 4

$\mathrm{cm}$ Petri dishes and 15-20 $\mathrm{ml}$ of pre-solidifying Sabouraud dextrose agar medium [10] were added to each plate. The plates were shacked slowly clockwise and anticlockwise to ensure homogenous distribution of the specimens in the agar media. The plates were transferred into sterilized plastic pages and incubated on $37 \pm 2^{\circ} \mathrm{C}$ to distinguish opportunistic Aspergillus spp from other nonpathogenic environmental molds for 7-10 days [11].

4. Blood samples: One $\mathrm{ml}$ of blood sample was inoculated into triplicate sterilized Petri dishes containg pre-solidified $15-20 \mathrm{ml}$ of Sabouraud dextrose agar medium. The plates were incubated on $37 \pm$ $2^{\circ} \mathrm{C}$ for $7-10$ days [12].

5. Biopsy: Biopsy sample was inoculated into pre-solidified Sabouraud's agar plates. Fungal growth was recorded after four days at $37 \pm 2^{\circ} \mathrm{C}$. Plates with no growth after 7-10 days were considered sterile [13].

6. ELISA techniques: Uncentrifuged BAL and serum specimens were sent to El-Mokhtabar lab for galactomannan (GM) and (1-3)- $\beta$ D-glucan (BDG) enzyme immunoassay according to the manufacture's specifications (Platelia Aspergillus, Bio-Rad, Marnes la Coquette, France) [14]. GM and BDG enzyme immunoassay test results were interpreted as positive when an optical density index of greater than or equal to 0.5 [15].

\section{B. Exhalation air fungal culture technique:}

Three Petri dishes containg Sabouraud dextrose agar medium in sterilized plastic pages opened and inverted were exposed to the exhalation air of the tracheal tube of the ventilator of the ventilated patients and incubated on $37 \pm 2^{\circ} \mathrm{C}$ for 7-10 days. Number of colonies were estimated $/ \mathrm{cm}^{3}$ of air $/$ minute.

\section{Results and Discussion}

Aspergillosis is a serious infection which is difficult to diagnose due to the lack of definitive diagnostic criteria [16]. Number of methods for the diagnosis had been applied $[17,18]$.

Two hundred and twenty seven patients were screened for aspergillosis suspected fungal species with different conventional methods. According to these methods, the results showed the presence of four species of Aspergillus which were A. niger (EN2 KY 6095), $A$. fumigatus, A. flavus (EN1 KY 609) and A. terreus. Aspergillus niger was the dominant species representing $32 \%$ of the total isolates followed by $A$. fumigatus and A. flavus with almost the same percentage (29\%) and finally $A$. terreus came with the least appearance between isolates (10\%) (Figure 1). No significant differences in species appearance were noticed among the different conventional methods. Furthermore, sputum cultures weren't sensitive enough that gave only $27.3 \%$ positive cultures from the total specimens tested, while blood cultures gave $42.8 \%$ positive cultures and the bronchoalveolar lavage gives $75 \%$ positive cultures (Table 1). False negative or positive results might have been occurred in one or more of these methods. Fungal infections are undoubtedly rare causes of pleural effusions but it is still important to recognize them [19]. Only one patient was suspected with aspergillosis during our study period and had pleural effusions. Culture of his pleural fluid reflects a fungal infection with $A$. niger and $A$. fumigatus and the patient had a dramatic improvement after antifungal treatment depending upon our fungal culture results. Forty one samples of serum and eight of BAL samples which were subjected to conventional culture technique in this study and proved to be positive cultures were subjected to ELISA technique for both GM and BDG antigens. The results confirmed the results of cultures with $75 \%$ positive in BAL galactomannan and $51.21 \%$ and $60.97 \%$ positive in serum GM and BDG, respectively which in power with our results (Table 1). These results revealed that BAL galactomannan gave higher rate of positive results than serum galactomannan while no statistical differences were recorded between galactomannan and BDG in serum.

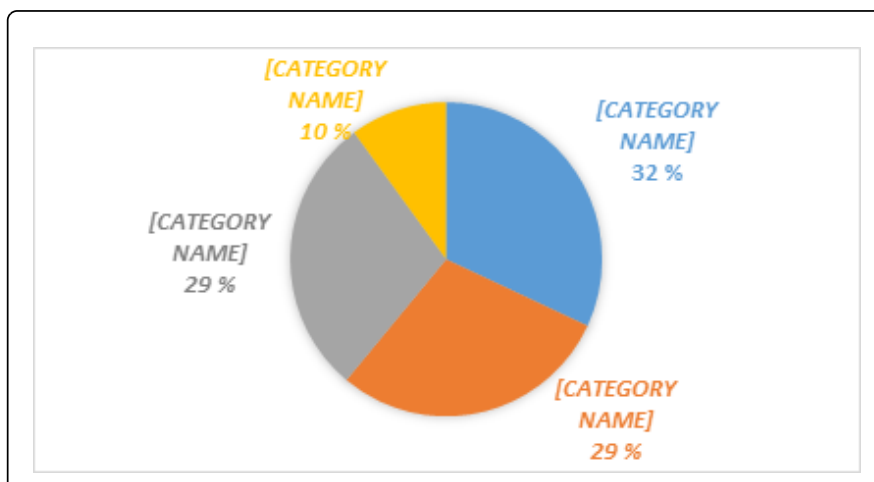

Figure 1: Percentage of fungal isolates appearance of aspergillosis survey from aspergillosis suspected patients suspected using conventional methods.

\begin{tabular}{|c|c|c|c|c|c|c|c|c|c|c|c|c|c|c|c|c|}
\hline & \multicolumn{10}{|c|}{ Culture } & \multicolumn{6}{|c|}{ ELISA technique } \\
\hline & \multicolumn{2}{|c|}{ Sputum } & \multicolumn{2}{|c|}{ Blood } & \multicolumn{2}{|c|}{ BAL } & \multicolumn{2}{|c|}{ Pleural fluid } & \multicolumn{2}{|c|}{ Biopsy } & \multicolumn{2}{|c|}{ BAL GM } & \multicolumn{2}{|c|}{ Serum GM } & \multicolumn{2}{|c|}{$\begin{array}{l}\text { Serum (1-3)- } \beta-D- \\
\text { glucan }\end{array}$} \\
\hline & No. & $\%$ & No. & $\%$ & No. & $\%$ & No. & $\%$ & No. & $\%$ & No. & $\%$ & No. & $\%$ & No. & $\%$ \\
\hline Positive & 41 & 27 & 21 & 43 & 8 & 32 & 1 & 100 & 1 & 50 & 6 & 75 & 21 & 51 & 25 & 61 \\
\hline Negative & 109 & 73 & 28 & 47 & 25 & 68 & 0 & 0 & 1 & 50 & 2 & 25 & 20 & 49 & 16 & 30 \\
\hline Total & 150 & 100 & 49 & 100 & 33 & 100 & 1 & 100 & 2 & 100 & 8 & 100 & 41 & 100 & 41 & 100 \\
\hline
\end{tabular}

Table 1: Percentage of positive fungal specimens from aspergillosis suspected patients using conventional methods.

Still identification in culture is the gold standard for the diagnosing of aspergillosis (simple and low cost) but may not be feasible due to the long time required and difficulty in obtaining samples in some cases of patients, lack of sensitivity and the difficulty in distinguishing between infection and colonization [20]. Also, sputum specimens are often contaminated with upper respiratory tract and mouth biota. 
Additionally young children are unable to reliably expectorate (cough up) sputum [21]. For these reasons Robert et al. [22] concluded in their study that the use of BAL to identify potential pathogens in the lower respiratory tract of adult patients is usually well tolerated and provides useful information. Radha et al. [23] confirmed the advantages of using of BAL as an accurate and sensitive diagnostic tool for aspergillosis. Blood cultures were effective only in cases of fungemia. It is supposed that many cases of invasive mycoses remain undiagnosed, mostly because of the insufficient sensitivity of blood cultures, which has been estimated as 50\% [24]. In contrast with Nawrot et al. [25] who showed that the culture of fungi from normally sterile tissue, including blood, remains an essential diagnostic test for invasive mycoses andthe addition of a mycosis medium to the standard set of aerobic and anaerobic vials improves the sensitivity of the detection of fungi and reduces the detection time and a growing number of fungemia cases have been observed in recent decades. Shuwei et al. [26] said that nearly a quarter of fungemia may be missed if a fungal blood culture is not performed. The early detection of diagnostic markers of a fungal infection, such as fungal nucleic acids, antigens, antibodies or cell wall components, is essential. For example, galactomannan antigen (GM) is widely used in the diagnosis of invasive aspergillosis. Another serum marker for the presence of invasive aspergillosis is (1-3)- $\beta$-D-Glucan (BDG), which has been included in the relevant diagnostic criteria of the European Organization for Research and Treatment of Cancer/ Mycoses Study Group [27]. Galactomannan (GM) is a polysaccharide Galactomannan, a major component of the Aspergillus cell wall, is released during invasive disease, and the level of circulating serum or plasma GM is indicative of the fungal burden in the host. This antigen serves as a biomarker of Aspergillus disease [28]. It has been documented that serum GM can be detected at approximately 7-14 days before other diagnostic clues become apparent, and therefore, monitoring of GM may allow initiation of preemptive antifungal therapy before establishment of the corresponding life-threatening infection [29]. The GM assay appears to be more specific but may be less effective during the early stage of infection [27]. Detection of GM in bronchoalveolar lavage (BAL) fluid has been advocated as a sensitive test for diagnosing of invasive aspergillosis, both in hematology and non-hematology patients, including solid-organ transplant recipients and critically ill patients.probabilities [30]. The polysaccharide BDG is a component of the cell wall of many fungi. Measuring the serum or plasma BDG levels has a high level of accuracy in the discrimination of patients with and without invasive fungal infections, mainly invasive fungal infections due to Candida or Aspergillus. The use of the BDG assay in combination with the GM assay could be of great interest to clinicians who can use these assays to exclude or confirm suspected invasive fungal infections [31]. Positive results of serology support diagnosis of invasive aspergillosis, although those should be remarked in conjunction with other diagnostic procedures, including microbiologic culturing, histopathological examination, and radiographical testing [32].

Ventilated patients suspected with aspergillosis are difficult to be diagnosed in intensive care units specially patients with an endotracheal tube or a tracheostomy as it is associated with high mortality and morbidity rates. Bronchoscopy technique for ventilated patients is invasive, involves risks and has its limitations in patients with severe pulmonary disease, high respiratory support settings and coagulation abnormalities. Facing a rapid emergence and dissemination of multi-drug resistant microorganisms particularly in the ICU environment, strategies to reduce such general and nontargeted antifungal consumption have become very important. For all the above obstacles in aspergillosis suspected patients with ventilation in ICU units we developed an easy method for diagnosis performance at the bedside, relatively simple, minimally invasive and inexpensive, have a proven acceptable accuracy and require minimal investment for the training of health professionals. Our method was culturing the exhaled air of the tracheal tube of the ventilator of ventilated patients and estimating the fungal causing agent of the disease. The results of twenty three of suspected aspergillosis patients tested proved eleven positive patients out of $23(47.8 \%)$ whereas the rest 12 were void out of any growth of aspergilli (Table 2). Aspergilli total count in positive exhalation air cultures were found to be ranged between 2.42 to 0.71 fungal colony $/ \mathrm{cm}^{3}$ air/minute. Figure 2 showed that the dominant fungal species was $A$. niger (48\%), followed by $A$. fumigatus (25\%), $A$. flavus (17\%) and finally $A$. terreus (10\%) which came in accordance with the results of conventional methods of the 227 patients tested. Moreover serology of GM and BDG in serum powered the results of the culture of exhalation of ventilation air that $65.21 \%$ positive in GM and $73.91 \%$ positive in BDG. Although, there are four and six false positive results recorded in GM and BDG, however the BDG could indicate fungal infections other than Aspergillus (Table 2). These results showed that collecting exhaled aerosols is technically simple to perform and has the potential to change perspectives of diagnosis and therefore improves strategies for the prevention and transmission of fungal respiratory diseases.

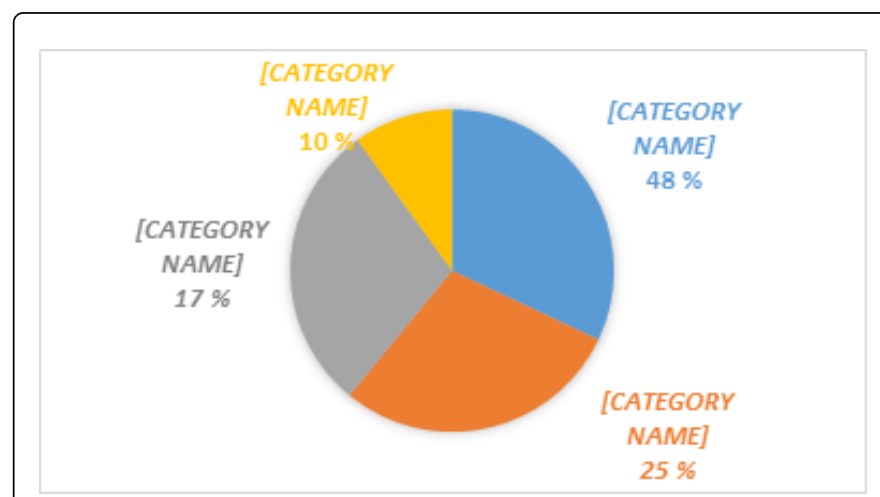

Figure 2: Percentage of fungal isolates appearance of aspergillosis survey from mechanically ventilated aspergillosis suspected patients suspected using exhalation air culture technique.

\begin{tabular}{|c|c|c|c|c|c|c|}
\hline & \multirow{2}{*}{\multicolumn{2}{|c|}{ Culture }} & \multicolumn{4}{|c|}{ Serology } \\
\hline & & & \multicolumn{2}{|c|}{ Serum GM } & \multicolumn{2}{|c|}{ (1-3)- $\beta$-D-glucan } \\
\hline & No. & $\%$ & No. & $\%$ & No. & $\%$ \\
\hline Positive & 11 & 48 & 15 & 65.2 & 17 & 73.9 \\
\hline Negative & 12 & 52 & 8 & 34.8 & 6 & 26.1 \\
\hline Total & 23 & 100 & 23 & 100 & 23 & 100 \\
\hline
\end{tabular}

Table 2: Percentage of positive fungal specimens from mechanically ventilated aspergillosis suspected patients using exhalation air culture technique. 


\section{Conclusion}

An accurate diagnosis of invasive aspergillosis is important and may improve patient survival and allow expensive and potentially toxic antifungal drugs to be withheld. Combining all traditional methods is important for the diagnosis. From all the conventional tools of the diagnosisno clear winner among them and an appropriate tool must be chosen according to the condition of the patient and the disease. Thus exhalation air culture is the clue of diagnosis of the ventilated suspected aspergillosis patients in ICU. It is easily performed at the bedside, relatively simple, minimally invasive and inexpensive and has a proven acceptable accuracy and requires minimal investment for the training of health professionals.

\section{References}

1. Biswas D, Agarwal S, Sindhwani G, Rawat J (2010) Fungal colonization in patients with chronic respiratory diseases from Himalayan region of India. Ann Clin Microbiol Antimicrob 9: 1-7.

2. Denning DW (1998) Invasive aspergillosis. Clin Infect Dis 26: 781-803.

3. Reichenberger F, Habicht JM, Gratwohl A, Tamm M (2002) Diagnosis and treatment of invasive pulmonary aspergillosis in neutropenic patients. Eur Respir J 19: 743-755.

4. Zmeili OS, Soubani AO (2007) Pulmonary aspergillosis: A clinical update. Medicine 100: 317-334.

5. Morgan J, Wannemuehler K, Marr K, Hadley S, Kontoyiannis D, et al. (2005) Incidence of invasive aspergillosis following hematopoietic stem cell and solid organ transplantation: interim results of a prospective multicenter surveillance program. Med Mycol 43: 49-58.

6. Chakrabarti A, Chatterjee SS, Shivaprakash MR (2008) Overview of opportunistic fungal infections in India. Nippon Ishinkin gakkai zasshi 49: 165-172.

7. Von Eiff M, Roos N, Schulten R, Hesse M, Zuhlsdorf M, et al. (1995) Pulmonary aspergillosis: early diagnosis improves survival. Respiration 62: 341-347.

8. De Carolis E, Posteraro B, Lass-Flo C, Vella A, Florio A, et al. (2012) Species identification of Aspergillus, Fusarium and Mucorales with direct surface analysis by matrix-assisted laser desorption ionization time-offlight mass spectrometry. Clin Microbiol Infect 18: 475-484.

9. Kuhlman JE, Fishman EK, Siegelman SS (1985) Invasive pulmonary aspergillosis in acute leukemia: characteristic findings on CT, the CT halo sign and the role of CT in early diagnosis. Radiology 157: 611-614.

10. Ronald MA (2000) Hand book of Microbiological Media (10thedn). CRC press, Inc. USA, pp: 137,333,785,279.

11. Takayoshi T, Koichi I, Masato T, Takahiro T, Yoshitomo M, et al. (2011) Diagnostic significance of Aspergillus species isolated from respiratory samples in an adult pneumology ward. Med Mycol 49: 581-587.

12. El-Ebiary M, Torres A, Fabregas N, de la Bellacasa JP, Gonzalez J, et al. (1997) Significance of the isolation of Candida species from respiratory samples in critically ill, non-neutropenic patients. An immediate postmortem histologic study. Am J Respir Crit Care Med 156: 583-590.

13. Zimmerli S, Urspeter K, Stephen L (2007) A model of cerebral aspergillosis in non-immunosuppressed nursing rats. Acta Neuropathologica 4: 404-411.

14. Sulahian A, Tabouret M, Ribaud P, Sarfati J, Gluckman E, et al. (1996) Comparison of an enzyme immunoassay and latex agglutination test for detection of galactomannan in the diagnosis of invasive aspergillosis. Eur J Clin Microbiol Infect Dis 15: 139-145.

15. Posteraro B, Torelli R, De Carolis E, Posteraro P, Sanguinetti M (2011) Update on the laboratory diagnosis of invasive fungal infections. Mediterr J Hematol Infect Dis 3: 201-1002.

16. Meersseman W, Vandecasteele SJ, Wilmer A, Verbeken E, Peetermans WE, et al. (2004) Invasive aspergillosis in critically ill patients without malignancy. Am J Respir Crit Care Med 170: 621-625.

17. Lass-Florl C (2009) The changing face of epidemiology of invasive fungal disease in Europe. Mycoses 52: 197-205.

18. Bajwa SJ, Kulshrestha A (2013) Fungal infections in intensive care unit: challenges in diagnosis and management. Ann Med Health Sci Res 3: 238-244.

19. Amber D, Richard W, Light M (2015) Pleural effusions. Infect Dis 8: 22-34.

20. Richard C (2013) Laboratory diagnosis of invasive aspergillosis: From diagnosis to prediction of outcome. Scientifica 2013: 1-19.

21. Antoniou M, Grossman RF (1995) Etiological diagnosis of pneumonia: A goal worth pursuing? Can J Infect Dis 6: 281-283

22. Robert P, Derborah A, Carlos P, Robert W (1997) Use of bronchoalveolar lavage semi quantitative cultures in cystic fibrosis. Am J Respir Crit Care Med 156: 286-291.

23. Radha S, Afroz T, Prasad S, Ravindra N (2014) Diagnostic utility of bronchoalveolar lavage. J Cytol 31: 136-138.

24. Cuenca-Estrella M, Verweij PE, Arendrup MC (2012) ESCMID guideline for the diagnosis and management of Candida diseases diagnostic procedures. Clin Microbiol Infect 18: 9-18.

25. Nawrot U, Kowalska-Krochmal B, Sulik-Tyszka B, Kozak M, Świętek K, et al. (2015) Evaluation of blood culture media for the detection of fungi. Eur J Clin Microbiol Infect Dis 34: 161-167.

26. Shuwei Z, Tong Y, Huihua L, Ai L, Thuan T, et al. (2016) A dedicated fungal culture medium is useful in the diagnosis of fungemia: a retrospective cross-sectional study. PLoS one 11: 1-15.

27. Fontana C, Gaziano R, Favaro M, Casalinuovo I, Pistoia E, et al. (2012) (1-3)- $\beta$-D-Glucan vs galactomannan antigen in diagnosing invasive fungal infections (IFIs). Open Microbiol J 6: 70-73.

28. Fisher CE, Stevens AM, Leisenring W, Pergam SA, Boeckh M, et al. (2013) The serum galactomannan index predicts mortality in hematopoietic stem cell transplant recipients with invasive aspergillosis. Clin Infect Dis 57: 1001-1004.

29. Cummings JR, Jamison GR, Boudreaux JW, Howles MJ, Walsh TJ, et al. (2007) Cross-reactivity of non-Aspergillus fungal species in the Aspergillus galactomannan enzyme immunoassay. Diagn Microbiol Infect Dis 59: 113-115.

30. Jorien D, Koen T, Edith V, Helene S, Greet D, et al. (2012) Detection of galactomannan in bronchoalveolar lavage fluid samples of patients at risk for invasive pulmonary aspergillosis: analytical and clinical validity. J Clin Microbiol 50: 1258-1263.

31. Racil Z, Kocmanova I, Lengerova M (2010) Difficulties in using 1,3- beta$\mathrm{D}$ glucan as the screening test for the early diagnosis of invasive fungal diseases in patients with hematological malignancies-high frequency of false positive results and their analysis. J Med Microbiol 59: 1016-1022.

32. Taghavi M, Mortaz E, Nikaein D, Athari SS (2015) Significance of galactomannan antigen for aspergillosis diagnosis: A review. Mycol Res 2: 111-117. 